\title{
CORRIGENDUM
}

\section{A histone lysine methyltransferase activated by non-canonical Wnt signalling supresses PPAR $-\gamma$ transactivation}

Ichiro Takada, Masatomo Mihara, Miyuki Suzawa, Fumiaki Ohtake, Shinji Kobayashi, Mamoru Igarashi, Min-Young Youn, Ken-ichi Takeyama, Takashi Nakamura, Yoshihiro Mezaki, Shinichiro Takezawa, Yoshiko Yogiashi, Hirochika Kitagawa, Gen Yamada, Shinji Takada, Yasuhiro Minami, Hiroshi Shibuya, Kunihiro Matsumoto \& Shigeaki Kato

Nat. Cell Biol. 9, 1273-1285 (2007); published online 21st October 2007

In the version of this Article initially published, Figs $6 a$ and $6 c$ contained panels that were inadvertently duplicated. In Fig. 6a, the panels in the right-hand column (Distal) corresponding to aSETDB1, aCHD7 and $\alpha$ NLK were duplicated. In Fig $6 c$, the panels in both the left (Proximal) and right (Distal) columns corresponding to $\alpha \mathrm{H} 3 \mathrm{~K} 9 \mathrm{me} 2$, $\alpha \mathrm{H} 3 \mathrm{~K} 9 \mathrm{me} 3$ and $\alpha \mathrm{SETDB} 1$ were duplicated. The correct versions of Figs $6 \mathrm{a}$ and $6 \mathrm{c}$ are provided below. The original conclusions of the study are unchanged.

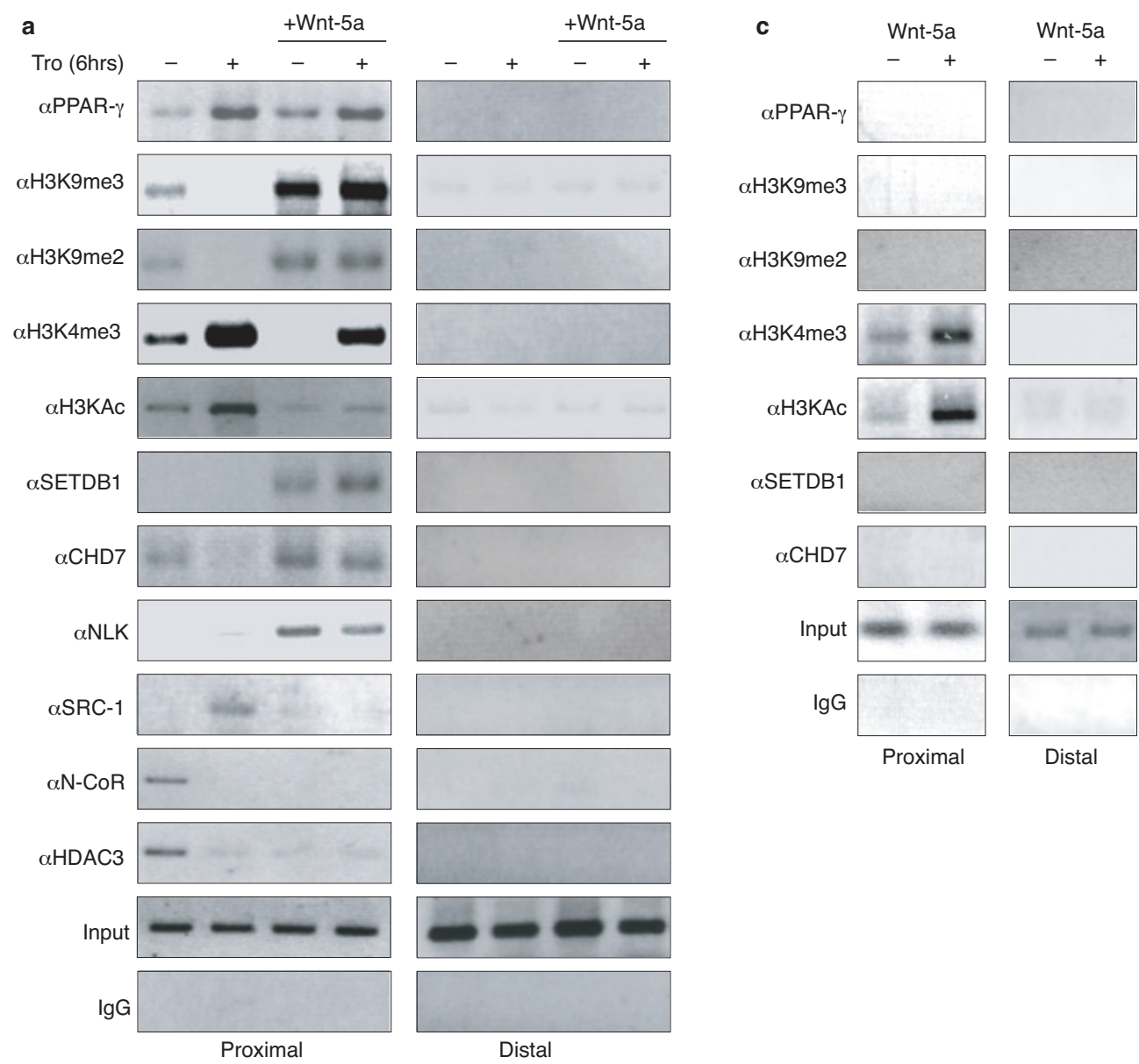

